\title{
DIE SYNOPSIS DES BARANYAI DECSI JÁNOS*
}

\author{
Zoltán ROKAY \\ Péter Pázmány Katholische Universität \\ H-1053 Budapest, Veres Pálné u. 24., Ungarn
}

\begin{abstract}
The Synopsis of János Baranyai Decsi - János Baranyai Decsi defended his theses on Aristotelian philosophy in Strasbourg, probably in 1590. The examining president was Johannes Ludovicus Hawenreuter. The "Synopsis Philosophiae..." of Decsi is presumably his series of examination theses ("Thesarium"). It is shows many parallels with Hawenreuther's textbooks on the same subject. In the Politics section of the Synopsis we find a number of alliterations and assonances reminiscent of the style of Sallust. Decsi was the first to translate Sallust into Hungarian. These figures may have been the individual contribution of the author who had an interest in aphorisms and adages.
\end{abstract}

Keywords: Synopsis, Aristotle, Hawenreuther, adagia, aphorism, János Erdélyi, Sallust, examination theses, Baranyai Decsi

Im folgenden Vortrag sollen einige Informationen und Vermutungen über die Synopsis Philosophiae in privatum memoriae studium thesibus et velut aphorismis quibusdam comprehensa, \& ad disputandum proposita in Academia Argentiniensi: Praeside Ioanne Ludovico Havvenreutero, Medicinae et Philosophiae Doctore atque professore. Respondente \& Authore Ioanne Deczio Ungaro erörtert werden.

In lateinischer Fassung liegt die Synopsis in zwei - uns bekannten Versionen vor (bis zur Abfassung des Vortrags):

1. Die Straßburger Version: Argentorati. Excudebat Antonius Bertramus.

(1591) und

2. die Wittenberger Version: Wittebbergae. Per praelum Welacianum. Anno 1595.

Weil die Straßburger Version mit kleineren Buchstaben gedruckt ist, umfaßt sie weniger Seiten als die Wittenberger. - Die Zeilen: „Haec studia adolescentiam alunt, senectutem oblectant etc.“ und „Iuventutis nutricula ...“ etc., befinden sich in der Straßburger Ausgabe an der Spitze, während sie in der Wittenberger Ausgabe am Schluß stehen mit der folgenden Bemerkung: „Haec duo elogia philosophiae cum non ferret pagella prima, ad calcem sunt reiecta."

* Es sei an dieser Stelle der Besatzung der Abteilung „Régi Nyomtatványok Tára“ der Országos Széchényi Könyvtár (Széchényi Nationalbibliothek) gedankt. Ohne ihre vielseitige Hilfe wäre dieser Vortrag nicht zustandegekommen.

1 Wittenberger Ausgabe, am Schluß, ohne Seitennummer. 


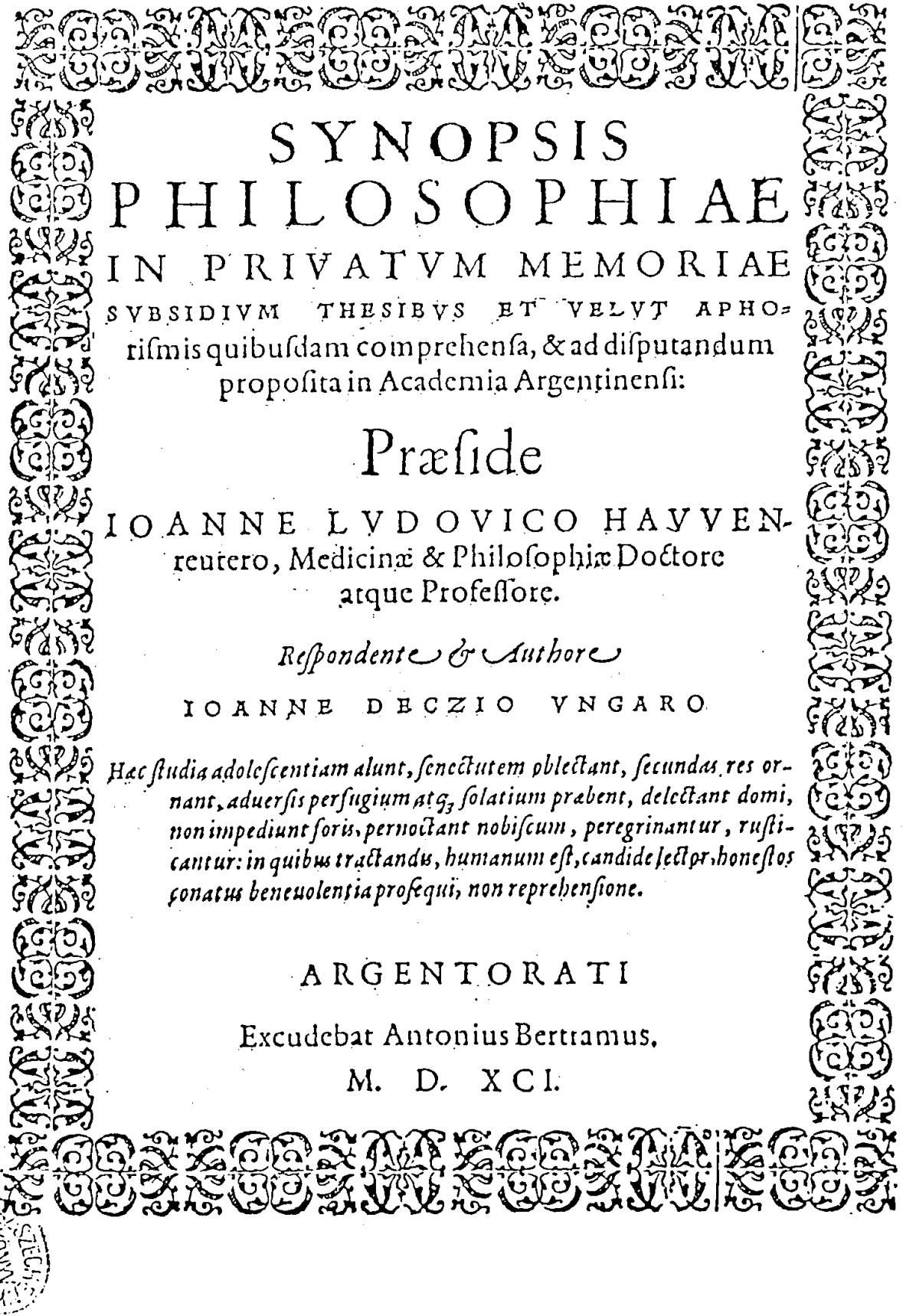

Abb. 1. Titelblatt von Synopsis Philosophiae (1591) von Baranyai Decsi 
Sonst konnte ich abgesehen von den wenigen Druck- und Lesefehlern, die außer „alten“ vier Fehlern auf das Konto des Neudrucks von Wittenberg gehen, keine wesentliche Abweichungen feststellen (z.B.: libros statt liberos, varietas statt veritas).

Die uns bekannten Exemplare der Synopsis befinden sich:

2 Wittenberger in der Széchényi Nationalbibliothek, Budapest.

1 Straßburger in Prag (Narodní Knihovna České republiky - Klementinum) ${ }^{2}$

1 Straßburger in Straßburg

1 Wittenberger in Wien ${ }^{3}$

Das Schicksal des von GÖMÖRI erwähnten Exemplars der Milich-Bibliothek zu Görlitz ist im Augenblick ungeklärt. ${ }^{4}$

Die kolossale Entdeckung und der große Verdienst von György GöMÖRI ist, daß er uns in seinem Aufsatz von ungarischen Autoren in England am Ende des XVI. Jahrhunderts auf die englische Übersetzung der Synopsis aufmerksam macht. Der englische Titel lautet: „A breife veiw (sic) of philosophy compiled in certaine positions and as it were aphorisms for the private helps of memory and propounded for disputation in the University of Argentinum“. Die Übersetzung ist uns handschriftlich erhalten. Als Datum des Abschlußes gilt der 13. September 1615. Sie befindet sich in der British Library, unter Signatur: Harley 405 folio 42-61. ${ }^{5}$

So wie damals GÖMÖRI die englische Übersetzung mit der Wittenberger Ausgabe nicht vergleichen konnte, so war ich nicht im Stande bis zur Abfassung des Vortrags das umgekehrte zu leisten. Die Divergenzen zwischen Straßburger und Wittenberger Ausgabe wiesen sich als unwesentlich aus. Es würde dementsprechend genügen eine von den obengenannten mit der englischen Übersetzung zu vergleichen. Sollten sich dabei bedeutende Abweichungen zeigen, so sollte man weiterhin eine weitere, uns unbekannte Ausgabe postulieren.

\section{INHALT UND AUFBAU - STIL UND FORM DER SYNOPSIS}

Die Aufteilung folgt der entsprechenden Aristotelischen Aufteilung der Wissenschaften in theoretische, praktische und poetische ; von den theoretischen behandelt sie die Mathematik, die Physik und die Metaphysik; von den praktischen die Ethik, Politik und die Oekonomie. (Wie er selbst - im Sinne des Aristoteles - ad Metaph. Nr. 81. sagt: „tria sunt autem genera scientiarum speculativarum, videlicet Mathematica, Physica et Metaphysica...").

Die Synopsis ist keine Aristoteles-Übersetzung, obwohl sie viele griechische Termini aus den aristotelischen Schriften bringt, mehrere Reminiszenzen auf ihn

\footnotetext{
${ }^{2}$ Mikrofilm in der Széchényi Nationalbibliothek, Budapest.

${ }^{3}$ Die beiden letzten Titeln aus: Index Aureliensis 1996, Baden-Baden, Prima Pars, Tomus XI.

${ }^{4}$ GÖMÖRI, György, Magyar szerzők múvei a XVI. század végi Angliában. (Ungarische Werke in England am Ende des XVI. Jhs.) In: Magyar Könyvszemle, 1980. No. 4. (Jhg. 96.) S. 392 ff.

${ }^{5}$ Ebd. S. 393.
} 


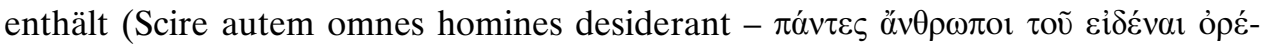

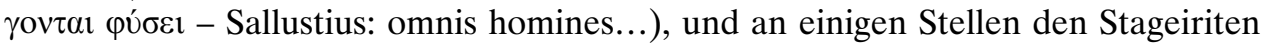
beim Namen nennt - was aber sonst nicht nötig ist; es wird irgendwie als selbstverständlich vorausgesetzt, daß es um seine Philosophie geht. - Doch einige Stellen: "id, quod Aristoteles ignoravit, nos ex revelatione, Dei sapientia scimus et credimus“ (Phys. Nr. 36); „ut vero haec tota Physices doctrina perfectior esset: historias animalium Aristoteles libris X, partes IV, generationem V comprehendit ..." (Ibid. Nr. $161)$; „Omnes enim in ore habent nobilitatem [inquit philosophorum princeps] cum vere nobiles vix centum reperiantur.“ (Polit. Nr. 48).

Es sei gleich auch auf die Stellen hingewiesen, die Platon nennen, oder sich auf ihn beziehen: „Sensibiles autem substantiae nequaquam generantur ab ideis, sicut Platon dixisse videtur...“ (Metaph. Nr. 90); - „Ideae vero Platonis nec sunt, nec quidquam iuvant..." (Ibid. Nr. 138); „Summum autem bonum nec consistit in corporis voluptatibus ... nec in ideis Platonicis, quae nullae sunt ..." (Ethica 3); „Plato has politiarum materias sustulisse videtur, cum coinoniam uxorum, liberorum ... aliaque nonnullae praeter rectam rationem intulit“ (Polit. Nr. 13); - „ideae non existunt“ (Metaph. Nr. 18); „nullae ideae possunt esse separabiles substantiis“ (Ibid. Nr. 95).

Die ganze Synopsis ist in Thesen und Definitionen abgefaßt. Es sei ein einziges Beispiel gebracht:

„Terraemotus est exhalatio calida \& sicca terrae visceribus inclusa, \& exitum quaerens, quem si ob soliditatem terrae non invenit eam movere solet." (Physica. Nr. 99).

Wie der Titel selbst sagt: sie ist eine Zusammenfassung. Am Rand des Textes befinden sich jeweils stichwortartige Angaben des Inhaltes, des eben zu behandelnden Satzes. Die Begründung dieser ganzen Abkürzung ist in der Einleitung angegeben: ars longa vita brevis!

In dem Teil über die Politik springen einige, an den Stil des Sallust erinnernde Alliterationen ins Auge:

Paucorum potentia (Nr. 59)

militiae robor penes poucos esse (ibid.)

divitibus poenam, pauperibus mercedem (Nr. 60)

mercede pauperes, poena divites vocentur ad conciones (Nr. 58)

(die beiden letzten Stellen sind sogar chiastisch)

si praemia per se, poenas per alios distribuat (Nr. 58, Punct. 16)

censu creari; censeri civitatem (Nr. 59)

Assonanz: opulentia \& paupertas

multitudo \& paucitas (Nr. 28)

Decsi ist der erste Übersetzer Sallust's ins ungarische. Sallust's aphoristischer Stil und Vorliebe für knappe, sentenzenartige Formulierung konnte Decsi's Interesse für Aphorismen, Paroimien mitbestimmen. 


\section{J. L. HAWENREUTER - ALS VORSTEHER DER DISPUTATION}

„Praeside Joanne Ludovico Havvenreuthero" heißt es auf dem Titelblatt der Synopsis. Havvenreuther wurde am 1. August 1548 zu Straßburg geboren, als Sohn Sebald H., der Arzt und Philosoph war, selbst ein Schüler Melanchtons, gebürtig aus Nürnberg. Johannes H. studierte in Straßburg Philosophie und in Tübingen Medizin. Widmete sich dem Unterricht von Physik und Logik. Er ,pflegte sich bei Disputationen nie zu erzürnen, war fast nie krank." ${ }^{6}$ Seine Werke, die in unserem Kontext interessant sind: Adagia Classica: scholis argentinensibus digesta: 1573; Aristotelis florum illustriorum ex universa eius philosophia collectorum libri tres. 1585; Compendium

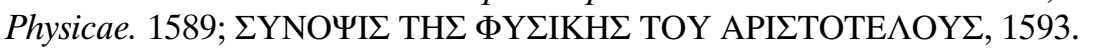

Für mich war nur dieses letzte Werk zugänglich. Es befindet sich in der Széchényi Nationalbibliothek, Budapest. Es ist in der Katechismusform (Frage-Antwort) gefaßt. Aus der Einleitung erfahren wir den Grund der Ausgabe: damit seine Schüler nicht „me invito excudendas traderent Typographo“ seine Vorlesungen. (So was könnte das Compendium Physicae aus dem Jahre 1589 sein). Wir finden Spuren seiner Arzneikunst in seiner Physik, u.a. in der Korrektur der Aristotelischen Auffassung, der „putavit nervos ex corde oriri.“ Havvenreuther war auch ein orthodoxer Gegner der Wiedertäufer. (Darüber spricht die Distinction, ob wir den Träumen die Möglichkeit der Zukunftsvorhersage zuschreiben sollen: In der Offenbarung ist das wohl möglich. Es ist auch möglich, daß Gott zuläßt, daß die unreinen Geister uns Träume sehen lassen; so: „....apud anabaptistas Monasterienses, qui somniis ad quaevis flagitia \& nefanda scelera monstruosissimasque opiniones incitati fuerunt.“ Synopsis, S. 579).

Das Verzeichnis der im deutschen Sprachbereich erschienenen Drucke des XVI. Jahrhunderts (VD 16) Bd.8, Nr. 818-841 evidentiert 16 Defendenten unter dem Vorsitz von Hawenreuther. Ihre Herkunft umspannt die geographische Weite von Flensburg bis Innsbruck. Von ihnen handelt einer über „Civitas Platonica“; von 15 übrigen erwähnt der Titel der Thesen bei 11 expressis verbis, bei 4 implicite Aristoteles oder seine Lehre.

Für uns sind interessant: Gallus Rhomanus Teccensis Transilvanus [über ihn siehe: Johann Jacob Grynaeus magyar kapcsolatai. (Ungarische Beziehungen des Johann Jacob Grynaeus) Szeged, 1989. S. 160-161] mit der These: Psychologia ... ex libris tribus Aristotelis. Disputiert am 28. XI. 1591 und: Fabinus Daniel Epperiensis

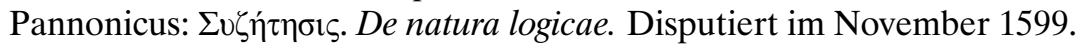

Es ist schwer zu beurteilen, in welchem Maße die Disputationsthesen eine eigenständige Leistung des Disputanden waren (abgesehen von der Bezeichnung: „Auctore“) - Neben Abweichungen (z.B. Hav.: Animus; Decsi: Anima) konnte ich innerhalb der Physik 36 teils wörtliche, teils sehr naheliegende Übereinstimmungen registrieren.

\footnotetext{
${ }^{6}$ Gelehrtenlexicon, z.St.
} 


\section{HAT JÁNOS ERDÉLYI DIE LITERARISCHE EIGENART DER SYNOPSIS IN SEINEM BUCH: DIE PHILOSOPHIE IN UNGARN, RICHTIG EINGESCHÄTZT?}

1. Erdélyi sagt nirgends, daß es um Disputationsthesen handelt.

2. Die Ablehnung Platons, sowie die Ausschließlichkeit des Aristoteles bei Decsi hängt meines Erachtens, vor allem mit den vorgelegten Thesen, mit dem Standpunkt des Aristoteles gegenüber Plato, sowie mit dem damaligen Schulwesen und Lehrplan zusammen.

Aristoteles, trotz der verbreiteten Meinung, die sich auf die Aussagen Luthers („Narristoteles“) und Melanchtons gründet („Quid enim ad nos, quid ille impurus homo commentus sit? Num Christo pluris Aristotelem faciemus?" $),{ }^{7}$ ist die aristotelische Philosophie die offizielle an den philosophischen Fakultäten, auch im protestantischen Bereich. - Melanchton selbst sagt: „,... ecclesiae opus esse liberali eruditione et non tantum cognitione grammatices, sed etiam aliarum multarum artium et philosophiae scientia“8 - „Die Theologie braucht die Philosophie, aber nicht irgendeine, sondern eine die möglichst wenig spitzfindig ist und die eine rechte Methodik bietet. ,Talis est Aristotelis doctrina“ ... Alle anderen philosophischen Schulen vertreten irgend etwas absurdes...".9

Johannes Sturm schreibt das Lob an den Melanchtonschüler Sebaldus Havvenreuther, den Vater von Johannes Ludovicus über seine lucubratiuncula: „Mihi vero perplacent: sunt enim Aristotelica: exclusis aliorum erroribus, eorumque refutationibus" (4. III. 1578). ${ }^{10}$ Hawenreuther selbst beruft sich sogar wo er über die Nützlichkeit der aristotelischen Physik spricht, auf die vielumstrittene Römerbriefstelle: „Die unsichtbare Wirklichkeit wird aus der sichtbaren wahrgenommen." (Röm I, 20). ${ }^{11}$

3. Es ist auch das kritisch zu beurteilen, was ERDÉLYI über die Beziehung Decsi's zur Religionslehre sagt: er weiche vom theologischen Denken weit ab, und läßt sie zugunsten der Philosophie weit hinter sich. ${ }^{12}$ Dazu scheint die Nennung von „D.O.M.“ - und was er über die Offenbarung sagt im Gegensatz zu stehen. (Deus Optimus Maximus; ,id quod Aristotelis ignoravit, nos ex revelatione, Dei sapientia scimus et credimus" - s. Phys. Nr. 46).

(Dieses ganze Fragekomplex könnte aber erst durch eine eingehende Studie über die Eigenart des Theologie- und Philosophiestudiums in Straßburg zur Zeit Hawenreuthers, bzw. Decsi's geklärt werden.)

\footnotetext{
${ }^{7}$ Melanchton - zitiert nach Heinz Scheible: Aristoteles und die Wittenberger Universitätsreform. In: Humanismus und Wittenberger Reformation. Leipzig, 1996. S. $141 \mathrm{f}$.

8 Ibid. S. 143.

9 Ibid.

${ }^{10}$ Sturm an Hawenreuthers Vater, in Hawenreuthers Synopsis, am Anfang, ohne Seitennummer.

${ }^{11}$ Römerbriefstelle in: Hawenreuthers Synopsis, in der Einführung über die Nützlichkeit der Physik, ohne Seitennummer.

12 ERDÉLYI, János, A bölcsészet Magyarországon (Geschichte der Philosophie in Ungarn). Franklin. Budapest 1885, S. 64-71, besonders S. 69 ff.
} 


\section{ZUSAMMENFASSUNG}

Auf Grund der bearbeiteten Unterlagen läßt sich m.E. folgendes sagen:

1. Die Synopsis ist eine Serie von Disputationsthesen; ${ }^{13}$

2. Sie ist abhängig von den Vorlesungen Hawenreuthers, als Vorstehers der Disputation.

3. Die Arbeit bei der Abfassung des Compendiums in der Form von Aphorismen könnte das Interesse Decsi's für Adagien-Literatur wecken; Hawenreuthers Adagia könnten ihm dabei als Vorbild dienen.

Weiteres und genaueres ließe sich über die Synopsis nur sagen, wenn man die übrigen Hawenreuther-Bücher und die Thesen der übrigen Straßburger Defendenten bei ihm unter Lupe genommen hat.

${ }^{13}$ Vgl. dazu: ZLINSZKY, János, Rezeption des römischen Rechts in Ungarn. in: Festgabe für Arnold Herdliczka. 1972. S. 320: „erwarb hier das Doktorat mit seinem Werke Synopsis..." u. ders.: Vita et opera Johannes Decii Barovii in: Publicationes Universitatis Miskolciensis, Fasc 1-4/1992. S. 208: ,ad doctoris gradum opere tituli Synopsis ...promotus est.“ 